\title{
Forecasting Rainfall based on Computational Intelligent Techniques
}

\author{
H. M. Abdul-Kader \\ Information Systems, Menufia \\ University, Cairo, Egypt
}

\author{
Ibrahim Selim \\ Computer Science Dept. Integrated \\ Thebes Institute, Cairo, Egypt \\ National Research Institute of \\ Astronomy and Geophysics Cairo, \\ Egypt
}

\author{
M. Abd-El Salam \\ Information Systems, Benha \\ University, Cairo, Egypt
}

\author{
A. Ahmad \\ Information Systems, Menufia University, Cairo, \\ Egypt
}

\author{
M. Mohamed \\ Information Systems, Higher Technological \\ Institute, Cairo, Egypt
}

\begin{abstract}
Forecast rainfall is a vital process to avoid hazardous causes from the climatic. So, the process of forecasting needs suitable technique has ability to treat with such problem and forecast rainfall accurately. This paper attempt to solve this problem through constructing Artificial Neural Network (ANN) especially Multi-Layer Perceptron (MLP) and applying two training algorithms on the constructed model (MLP) to train and test it. First training algorithm is an optimization algorithm which based on a global search Particle Swarm Optimization (PSO). Second training algorithm is another type of Back Propagation (BP) is Levenberg-Marquardt (LM). Comparing the model of MLP with two training algorithms with another model is Redial Basis Function (RBF). Applying RBF on the same weather data used on two training algorithms. The results approved that MLP based PSO is the most effective comparing with MLP based LM and RBF, through the error value of RMSE for each one.
\end{abstract}

\section{Keywords}

Forecasting the Weather, Feed Forward Neural Network, Levenberg-Marquardt algorithm, Multi-Layer Perceptron, Particle Swarm Optimization and Redial Basis Function.

\section{INTRODUCTION}

The state of weather considers the main factor in different fields as transportation, health, agriculture...etc. Forecasting the weather in future won the interest of many researchers in different countries around the world, especially forecasting rainfall. The rainfall intensely causes many problems as floods, which threat and dangerous for many things as damage agriculture, control in flight, threatens human...etc. The state of weather changes continually from period to another period. The forecasting need technique that has the ability to discover novel and potentially of useful information from large amounts of data.

These problems make forecasting a formidable challenge. Thus, it is important to make adequate forecasting through sufficient technique. Artificial Neural Network (ANN) consider more suitable technique than traditional technique. It has the ability to treat with dynamic and change of the weather because it has many benefits as extract meaning from complicated or imprecise data and learns how to perform a task-based on data provided to train instead of human [1]. Training constructed ANN-based on an optimization algorithm
Particle Swarm Optimization (PSO), instead of Back Propagation (BP) to forecast rainfall.

\section{TECHNIQUES USED \\ 2.1 FEEDFORWARDNEURAL NETWORK (FFNN)}

Feed Forward Neural Network (FFNN) is considered the popular kind of ANN. The data flows in unidirectional and may consists of the input layer and the output layer, this type called single layer. The network may consist of the input layer, one hidden layer, and output layer called Multi-Layer Perceptron (MLP) [2].

\subsection{Multi-Layerperceptron (MLP)}

MLP is the most common type used in FFNN known as a supervised neural network because it requires the desired output for learning. MLP is used to develop a model that use certain parameters of historical data as input to the output. MLP may be consisted of the input layer, one hidden layer, and output layer. The network may have more than one hidden layer. The actual output of the network which has more than one hidden layer is more accurate than the actual output of the network that has one hidden layer [3]; but the network which has more than one hidden layer takes a long time to train. Back Propagation (BP) is the most popular algorithm used to train MLP.

\subsection{Levenberg-Marquardt (LM)}

One of the most popular algorithm for nonlinear problems; LM is another type of BP training algorithm has been also used for ANN training. Although LM is more powerful than gradient descent techniques, it does not always guarantee global optimum for the problem [4].

\subsection{Redial Basis Function (RBF)}

The RBF network composed of three layers are Input layer, Hidden layer and Output layer as shown in Figure 4.6. It has only one hidden layer otherwise multilayer structure, which may has one or two hidden layer [5].

Training the network divided into two phases [6]:

First phase, the weights from the input to hidden layer is determined by computing the distance $\left(d_{i}\right)$ between the input vector $x$ and the center of basis function $c_{i}$ as shown in equations 1 and 2 .

$$
d_{i}=\left\|x-c_{i}\right\|
$$




$$
\varphi_{i}=\exp \left(-\frac{d_{i}}{2 \sigma^{2}}\right)
$$

Where $\varphi_{i}$ is Gaussian function for each node at hidden layer, $\sigma$ is spread or width for each node in hidden layer.

Second phase, the weights from the hidden to output layer as shown in following equation.

$$
y_{i k}=\sum_{i=1}^{n} w_{i k} \varphi_{i}
$$

Where $y_{i k}$ is output, $w_{i k}$ is weights between hidden layer and output layer.

\subsection{Particle Swarm Optimization (PSO)}

PSO developed by Kenndey and Eberhart 1995, a stochastic algorithm which its idea mimic behaviors of animals as flock of birds and fish schools [85]. A flock of birds doesn't has a leader. There is always a bird closest to the food source and this means that bird has optimistic position and other birds attempt to follow that bird where its position closest to the source of food[7]. In the PSO algorithm, each particle in population is reckoned as a solution that algorithm works on finding optimal values as work of bird flock and fish school. Each particle changes its velocity and its position according to equations 4 and 5:

$$
\begin{gathered}
V_{i}(t+1)=W V_{i}(t)+c_{1} r_{1}\left(P_{i}(t)-X_{i}(t)+c_{2} r_{2}\left(P_{g}(t)\right.\right. \\
-X_{i}(t)
\end{gathered}
$$

Where $W$ represent inertia weight, $V_{i}(t+1)$ represent new velocity of particle $\mathrm{i}$ in next iteration, $V_{i}$ represents velocity of particle $\mathrm{i}, \mathrm{t}$ represents an iteration number $c_{1}, c_{2}$ represent the learning rate for individual (local) and group (Global) $r_{1}, r_{2}$ are random values have values between [0-1], xi represents the current position of particle $\mathrm{i}, P_{i}$ symbolize the local best for particle $\mathrm{i}$, and $P_{g}$ represent the global best for the swarm.

$$
X_{i}(t+1)=X_{i}(t)+V_{i}(t+1)
$$

Where $X_{i}(t+1)$ represent the new position at next iteration.

On each iteration of the algorithm the current position considers as solution and if that position better than the previous according to its value of fitness function which has a minimum value (minimize problem), that position considers Pbest [8].

\section{PRPOSED MODEL}

Our proposed technique used for forecasting rainfall is represented into two steps as the following:-

First step: Develop ANN has the following features:-

- Input layer: has four nodes (neurons)

- One hidden layer: has 20 node (neuron).

- Output layer: has one node (neuron).

Second step: using an optimization algorithm based on stochastic search (PSO) instead of gradient descent (BP) to train the network as shown in figure 1, PSO avoids fall in a local minimum, because it is not based on gradient information

1. Initialize population size and maximum iterations for PSO.

2. Applied all particles to train constructed ANN and calculate fitness function (FF) (RMSE) to each particle in the population.

3. Determine personal best (Pbest) and global best (gbest) at each iteration which has the minimum value of RMSE.

4. Other particles update its velocity based on gbest as equation (4) and position as equation (5).

5. Repeat step two and calculate FFfor each particle; if current $\mathrm{FF}$ of the particle < pbest then current $\mathrm{FF}=$ pbest, otherwise pbest remain.

6. The pbest with the best value in all particles is set as gbest; after that repeat step four.

7. Repeat steps from two to six until reach maximum iterations or reach to zero.

8. After that, the best set of weights (particle position) has the lowest value of RMSE for the whole swarm becomes known and set as gbest.

9. After the training phase finished, testing phase starts with these weights used in the training phase to generate a final output of the network. 


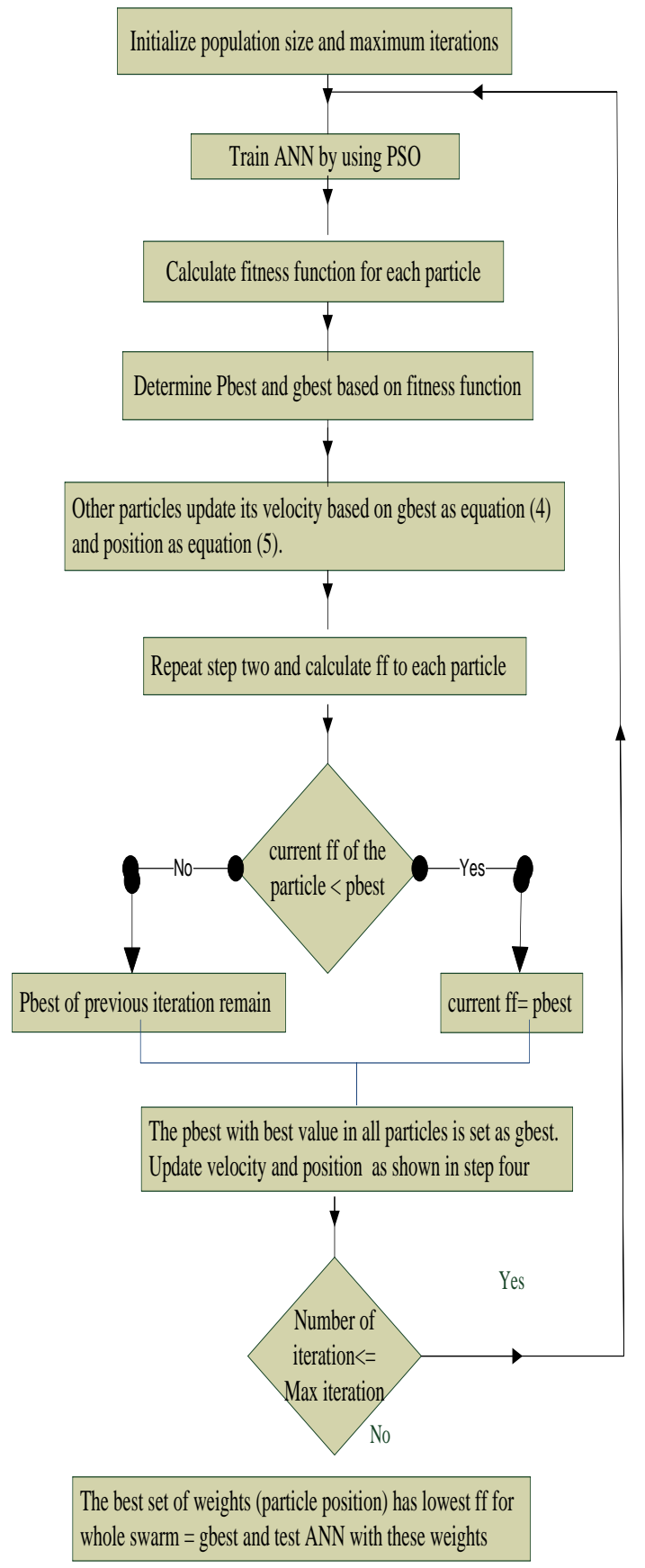

Fig1: Flowchart Algorithm

\section{EXPERMENTAL RESULTS AND DISCUSSION}

\subsection{Data Set}

We applied weather data of 2009 for Cairo City by three techniques (MLP based PSO), (MLP based LM), and RBF to forecast the rainfall. Through using law temperature, high temperature, humidity and wind speed as input parameters where rain rate is output parameter for three techniques.

\subsection{Result and Discussion}

\subsubsection{Comparison Our Constructed Model By \\ Two Algorithms}

i. Training Process

We classified data set into train and test, used $90 \%$ train and the remainder of data $(10 \%)$ is test. We applied two different algorithms (PSO and LM) to train our constructed model (MLP). Output of two training algorithms are demonstrated through Figure 2, where horizontal axis represent samples (measurements at every two hours for parameters of the weather), vertical axis represents values of rainfall where actual output (train class). The output of MLP based PSO is closer than the output of MLP based LM to train class.

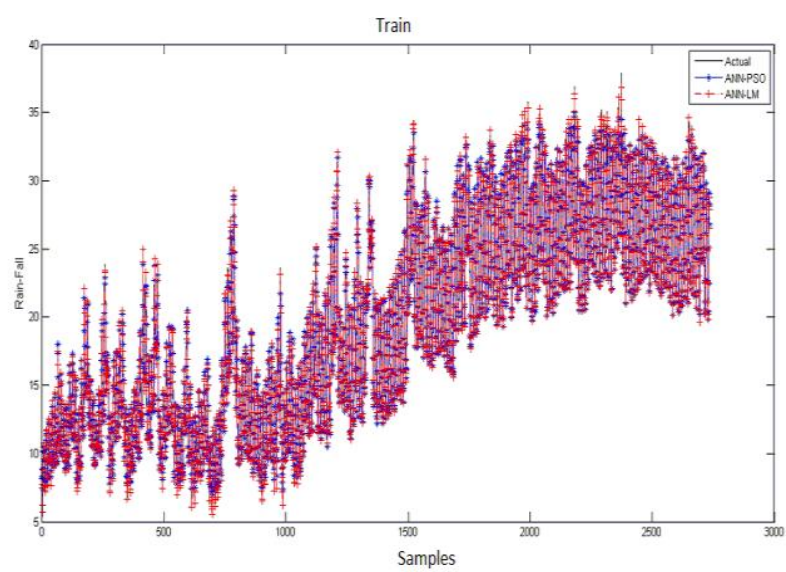

Fig 2: Training MLP Based two algorithms

Using statistical method to measure the performance of the network, through measuring the error of each technique. The statistical method is RMSE as shown in table one, where RMSE value for MLP Based PSO is less than RMSE value for MLP Based LM.

Table 1. The resulted Error from Training Two Algorithms

\begin{tabular}{|l|c|}
\hline Techniques & RMSE \\
\hline MLP Based PSO & 0.12 \\
\hline MLP Based LM & 0.15 \\
\hline
\end{tabular}

\section{ii. TESTING PROCESS}

Test the model through using the remainder of data set (10\%) which unseen data to test the efficiency of the model. The output of the two training algorithms are demonstrated through Figure 3. We observed that the output of MLP based PSO is closer than the output of MLP based LM to train class.

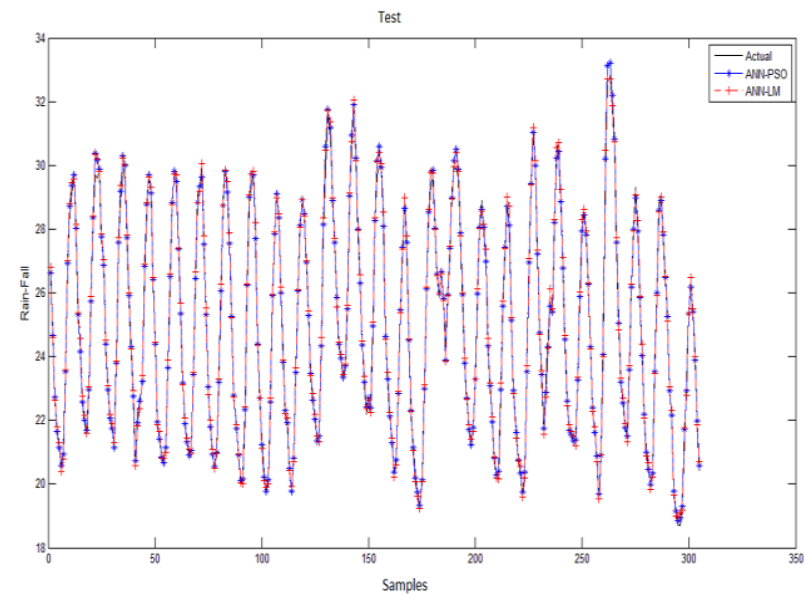

Fig 3: Testing MLP based two algorithms

MLP based PSO is more effective than MLP based LM to forecast rainfall, which approved through value of RMSE for two algorithms as shown in table two. 
Table 2. The resulted error from testing two algorithms

\begin{tabular}{|l|c|}
\hline Techniques & RMSE \\
\hline MLP Based PSO & 0.14 \\
\hline MLP Based LM & 0.18 \\
\hline
\end{tabular}

\subsubsection{Comparison with Other Model}

We applied our data set on another model is RBF and compare its result with two techniques (MLP-PSO) and (MLP- LM).

\section{i. $\quad$ Training Process}

We trained the independent variables (train set) to forecast the rainfall as shown in Figure 4, where output obtained from RBF is illustrated in red line whereas actual output (train class) illustrated in black line.

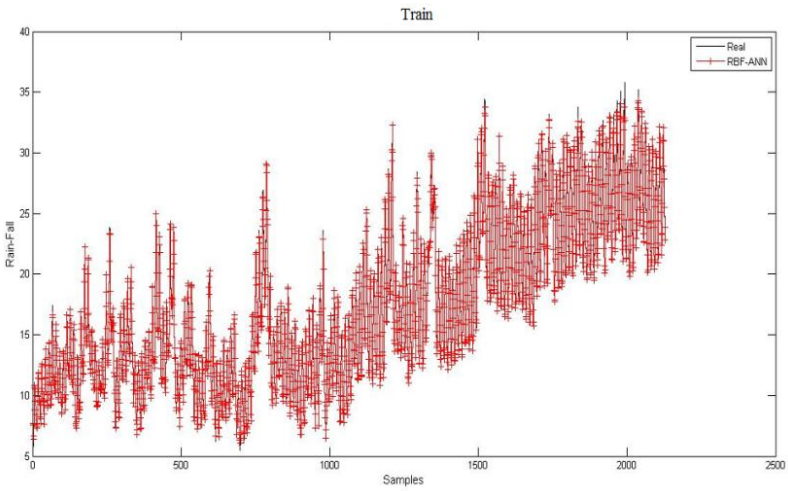

Fig4: Training RBF

\section{ii. TESTING PROCESS}

After training process is finished, the network has the ability to forecast rainfall. To guarantee its ability for generalization, we test the network using unseen data as shown in Figure 5.

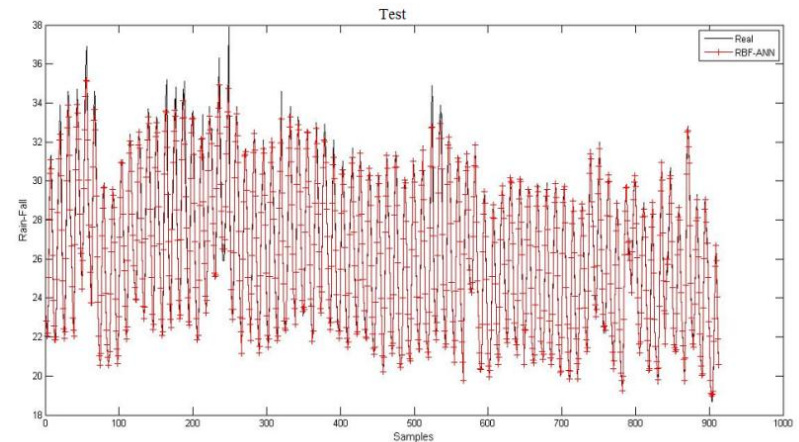

Fig5: Testing RBF

The output of RBF is compared with actual output and calculate the difference between them through RMSE as shown in table 3.

Table 3 RMSE for RBF

\begin{tabular}{|c|c|}
\hline Process & RMSE \\
\hline Training & 0.35 \\
\hline Testing & 0.44 \\
\hline
\end{tabular}

We observed through comparing three models (MLP-PSO, MLP-LM and RBF); MLP-PSO is the most efficient model for our problem as shown in table 4.

Table 4 comparison of three models

\begin{tabular}{|l|c|c|}
\hline Technique & Training & Testing \\
\hline MLP-PSO & 0.12 & 0.14 \\
\hline MLP-LM & 0.15 & 0.18 \\
\hline RBF & 0.35 & 0.44 \\
\hline
\end{tabular}

\section{CONCLUSION}

The hybrid technique approved its efficiency for forecasting the rainfall compared other techniques as MLP based LM, RBF through applying weather data of 2009 for Cairo City. We concluded that using (MLP) alone cannot provide an accurate result and PSO as training algorithm outperforms LM and RBF through measurement of RMSE for error.

\section{REFERENCES}

[1] Rahul Moriwal, and Shiv Kumar Dubey, "Predicting Weather Using Data Mining Techniques," International Journal of Advanced Engineering \&Global Technology (IJAEGT), Vol. 3, pp. 51-59, February 2012.

[2] Ratna Nayak, P.S. Patheja, and Akhilesh Waoo, "An Enhanced Approach for Weather Forecasting Using Neural Network," Proceedings of the International Conference on SocProS, Vol. 131, pp. 833-839, December 2011.

[3] Rahul Moriwal, and Shiv Kumar Dubey, "Predicting Weather Using Data Mining Techniques," International Journal of Advanced Engineering and Global Technology( IJAEGT), Vol. 3, No. 5,pp. 646-649, May 2015.

[4] Saduf, and Mohd Arif Wani," Comparative Study of Back Propagation Learning Algorithms for Neural Networks," International Journal of Advanced Research in Computer Science and Software Engineering (ijarcsse), Vol. 3, PP. 1151- 1156, December 2013. Available online at: www.ijarcsse.com.

[5] Yojna Arora, Abhishek Singhal, and Abhay Bansal, "A Study of Applications of RBF Network," International Journal of Computer Application (IJCA), Vol. 94, No. 2, PP. 17-20, May 2014.

[6] I. El-Feghi , Z. Zubia and S. Abozgaya , "Efficient Weather Forecasting using Artificial Neural Network as Function Approximator," international journal of neural networks and advanced applications, Vol. 1, pp. 49-55, 2014.

[7] Russell C. Eberhart, and Yuhui Shi, Particle Swarm Optimization Development, Applications and Resources," IEEE, 2001.

[8] Riccardo Poli, James Kennedy, and Tim Blackwell, "Particle swarm optimization An overview," Springer Science + Business Media, May 2007. 